\title{
Noncommutative de Sitter and FRW spaces
}

\author{
Maja Buricí ${ }^{1, a}$, John Madore 2,b $^{\text {,b }}$ \\ ${ }^{1}$ Faculty of Physics, University of Belgrade, P.O. Box 44, Belgrade 11001, Serbia \\ ${ }^{2}$ Laboratoire de Physique Théorique, 91405 Orsay, France
}

Received: 27 August 2015 / Accepted: 9 October 2015 / Published online: 24 October 2015

(c) The Author(s) 2015. This article is published with open access at Springerlink.com

\begin{abstract}
Several versions of fuzzy four-dimensional de Sitter space are constructed using the noncommutative frame formalism. Although all noncommutative spacetimes which are found have commutative de Sitter metric as a classical limit, the algebras and the differential calculi which define them have many differences, which we derive and discuss.
\end{abstract}

\section{Introduction}

Various and sundry reasons have been put forward to entice physicists into spacetime noncommutativity. It has for example been argued that noncommutative geometries could incorporate some aspects of the quantized gravitational field. There seems further to be no obvious physical reason to extrapolate the commutativity of coordinates and the corresponding description of spacetime as a manifold to arbitrarily small length scales. However, if one approaches the task of defining 'noncommutative space' from a physical or physically useful point of view and not as a purely mathematical abstraction, there is a long list of properties which one might like to incorporate in order to be able to use the standard language of dynamics and symmetries.

Inspired by the fact that the geometry of a smooth manifold can be described in terms of the algebra of smooth functions defined on it, probably the most plausible starting point is to define a noncommutative space as an algebra $\mathcal{A}$ of linear operators. This approach in many ways inherits intuition from quantum mechanics. There are other approaches which rest on insights and constructions from string theory or conformal field theory, [1-3]. Apart from spacetime, one always has field equations which involve usually the action of a Laplace or Dirac operator: one must that is define derivations. It is not a priori obvious which properties derivations should possess but a natural condition is the Leibniz rule;

\footnotetext{
a e-mail: majab@ipb.ac.rs

be-mail: madore@th.u-psud.fr
}

there are, however, important models in which differential operators do not obey it [4-6]. At the risk of narrowing down the class of possible structures, we attempt to extend the various elements of classical geometry to a simple and well studied structure such as algebra $\mathcal{A}$. One important physical aspect is symmetry: symmetries are in the algebraic framework represented quite natural. Finally, a relevant question which one has to address is that of the commutative or classical limit. Given that associative algebras are rigid structures constrained by the Jacobi identities, it is not clear whether it is possible to fulfill all these requirements in a physically or mathematically satisfactory way.

There are indications, both from quantum mechanics and general relativity, that when introducing a noncommutative space one should consider not just spacetime that is position space, but the full phase space. In quantum mechanics phase space consists of commutative coordinates $x^{\mu}$ and (here taken antihermitian) momenta $\hbar p_{\alpha}=\delta_{\alpha}^{\mu} \partial_{\mu}$. The adjoint action of momenta $p_{\alpha}$ on elements $f(x) \in \mathcal{A}$ of the position algebra defines the derivations,

$\left[p_{\alpha}, f\right]=\left(e_{\alpha} f\right)=\delta_{\alpha}^{\mu}\left(\partial_{\mu} f\right)$,

in particular

$\left[p_{\alpha}, x^{\mu}\right]=\delta_{\alpha}^{\mu}$.

This can be seen perhaps more clearly by using explicitly the Hilbert space $\mathcal{H}$ on which the representation of the algebra acts: derivations appear as momenta. In a completely analogous way one can interpret the frame derivations $e_{\alpha}$ defined in the Cartan frame description of geometry,

$e_{\alpha}=e_{\alpha}^{\mu}(x) \partial_{\mu}$,

as momenta conjugate to coordinates, again assuming the adjoint action on the functions of coordinates. The canonical commutators in the gravitational field change, 
$\left[p_{\alpha}, x^{\mu}\right]=e_{\alpha} x^{\mu}=e_{\alpha}^{\mu}(x)$,

and characterize the curved spacetime because the $e_{\alpha}$ do not in general commute,

$\left[e_{\alpha}, e_{\beta}\right]=C_{\alpha \beta}^{\gamma}(x) e_{\gamma}$.

The last relation can be written as a commutator of the momenta

$\left[p_{\alpha}, p_{\beta}\right]=C_{\alpha \beta}^{\gamma}(x) p_{\gamma}$

which acts in the adjoint representation on an arbitrary function $f(x)$. Quite naturally, noncommutativity in momentum space is equivalent to curvature in position space. By classical duality between position and momenta, noncommutativity in position space then is related to curvature in momentum space: we thus conjecture that curvature and noncommutativity are two aspects of the same reality.

We mentioned that it is not easy to extend all physical and geometrical requirements to noncommutative space. A particularly delicate question in noncommutative geometry is that of dimension. On a commutative manifold to describe a point we need $n$ real parameters; this integer is also the dimension of the tangent space as each vector $X$ can be expanded as

$X=X^{\mu}(x) \partial_{\mu}$.

The set of derivations $\{X\}$ is a left $\mathcal{A}$-module and the de Rham differential is uniquely defined. Phase space has $2 n$-dimensions.

Counting dimensions is different on noncommutative space and in order to obtain some intuition we point out the differences which appear in the simplest examples. Let us take the space $M_{2}$ of $2 \times 2$ (or $M_{n}$ of $n \times n$ ) complex matrices. As a linear space it has 4 complex, or 8 real dimensions. The subspace of hermitian matrices has 4 real dimensions, and a suitable basis is for example given by the Pauli matrices and unity, $\left\{\sigma^{i}, \mathbb{I}\right\}$. However, if we consider the set $M_{2}$ as an algebra, we need only two $\sigma$-matrices to generate it, for example $\sigma_{x}$ and $\sigma_{y}$, as $\sigma_{x}^{2}=\mathbb{I}, \sigma_{x} \sigma_{y}=i \sigma_{z}$. Therefore the number of generators of the algebra is 2 and one might conclude that its dimension is 2 as well. In a similar manner, the infinite-dimensional linear algebra of operators on the Hilbert space of quadratically integrable functions of one variable $x$ is generated by two operators, $x$ and $\partial_{x}$, or $a$ and $a^{\dagger}$.

If we consider, on the other hand, a set of inner derivations $\{X\}$ on matrix space $M_{2}$,

$X_{p} f=[p, f], \quad p \in M_{2}$, we see that it is of dimension 3 (that is, $n^{2}-1$ in $M_{n}$ ) because $[\mathbb{I}, f]=0$. On a noncommutative space the set of derivations is no longer a left module, that is, $f X$ is not a derivation along with $X$ because it does not satisfy the Leibniz rule. Therefore in order to determine dimension of the tangent space we have to 'count' momenta $p$ as a linear space over the real or complex numbers. Consequently, the dimension of the tangent space always differs from dimension (the number of generators) of the algebra itself. We see thus that the notion of dimension does not have a precise meaning and speaking of it we usually try to relate it to the commutative limit of a given noncommutative geometry. A further reason is that we wish to interpret a specific algebra as a noncommutative space independently of its representation, whereas linear dimension is related to dimensionality of representations.

The noncommutative frame formalism [7] solves this problem in the following manner. On $\mathcal{A}$ a differential $d$ can be defined for all vector fields in analogy with the commutative case,

$\mathrm{d} f(X)=X f$.

But as we have seen, the linear space of all vector fields is 'too big': we can redefine $d$ by restricting it on a subset $\left\{e_{\alpha}\right\}$ of the set of all derivations. Thereby we define the tangent space. Let $\left\{\theta^{\alpha}\right\}$ be the set of 1 -forms dual to $e_{\alpha}, \theta^{\alpha}\left(e_{\beta}\right)=\delta_{\beta}^{\alpha}$. Then a restriction of $d$ is defined by

$\mathrm{d} f=\left(e_{\alpha} f\right) \theta^{\alpha}$.

The set of 1-forms is a bimodule, that is, along with $\theta^{\alpha}, f \theta^{\alpha}$, and $\theta^{\alpha} f$ are 1 -forms. Duality is equivalent to the 'frame condition'

$\left[f, \theta^{\alpha}\right]=0, \quad \forall f \in \mathcal{A}$.

Vector fields can always be given as commutators (1.8), but momenta $p$ may not belong to position algebra $\mathcal{A}$. Coordinates and momenta together generate phase space. A peculiar property now is that dimension of phase space is in general not equal to $2 \times$ dimension of spacetime. This comes about because of noncommutativity of coordinates: for the Heisenberg algebra for example, that is, the two-dimensional Moyal space where

$[x, y]=i$,

we can take $p_{x}=i y, p_{y}=-i x$, and we get

$\left[p_{i}, x^{j}\right]=\delta_{i}^{j}$, 
the flat frame. In this case phase space is identical to position space. Similar situation we have for matrix algebras $M_{n}$ because on $M_{n}$ all derivations are inner. We thus see that in the noncommutative frame formalism by the choice of the set $\left\{e_{\alpha}\right\}$ we effectively fix the dimension of spacetime (defined as dimension of its tangent space). This choice is by no means unique and reflects the property that the differential $d$ on an algebra is not unique either.

An important characteristic of the noncommutative frame formalism is that, if momenta generate the same algebra as coordinates, that is, if all derivations are inner, they must satisfy a quadratic relation,

$2 P^{\alpha \beta}{ }_{\gamma \delta} p_{\alpha} p_{\beta}-F^{\beta}{ }_{\gamma \delta} p_{\beta}-K_{\beta \gamma}=0$,

where $P^{\alpha \beta}{ }_{\gamma \delta}, F^{\beta}{ }_{\gamma \delta}$, and $K_{\beta \gamma}$ are constants. This relation follows from stability of Eq. (1.11) under the differential,

$\mathrm{d} f=-[\theta, f], \quad \theta=-p_{\alpha} \theta^{\alpha}$,

and the constraint $d^{2}=0$. We stress the simplicity and importance of these equations as well as their content. On the one hand they define differential calculi on an arbitrary algebra in much the same way that the de Rham calculus is defined on a smooth manifold. On the other hand we see immediately that the calculus is not unique; each choice of the momenta consistent with (1.14) gives a different $d$. Finally, (1.11) allows one to interpret 1 -forms $\theta^{\alpha}$ as the moving frame assuming that the frame components of the metric are constants, for example

$g^{\alpha \beta}=\eta^{\alpha \beta}$.

In the commutative case there are no restrictions from the commutators or from the associativity of the product; momenta are necessarily external and from (1.5) we see that there is no analog of (1.14).

\section{Noncommutative de Sitter space, I}

We saw in the previous section that the frame formalism gives a definition of differential general enough to include commutative manifolds, quantum-mechanical phase space and noncommutative matrix spaces. Its main constituent is the moving frame which naturally incorporates geometry. To see whether this formalism can indeed describe noncommutative gravity we proceed by examples which fulfill previously mentioned requirements and have a certain relevance in physics. We discussed in previous papers [8-10] various rotationally invariant noncommutative spaces. In this paper we give examples of algebras with spherical symmetry which can be considered as fuzzy versions of cosmological metrics: de Sitter and Friedmann-Robertson-Walker. We find several versions of noncommutative four-dimensional de Sitter space which have different spectral and symmetry properties, but the same limiting classical metric. Another common feature which they share is that one needs to make some kind of dimensional extension to obtain a smooth noncommutative space.

Perhaps the most natural idea, when we think of constructing noncommutative de Sitter spacetime, is to start from the Lie algebra of the de Sitter group itself. This idea was in some detail put forward in $[11,12]$, as a generalization of the fuzzy sphere construction [13]: we shall in this section analyze and develop it. Let us briefly recall the fuzzy sphere. The possibility to interpret the $S O(3)$ group generators $x^{i}$, $i=1,2,3$, as Cartesian coordinates on the sphere is based on two facts. The first is that operators $x^{i}$ in the irreducible representations satisfy the Casimir relation,

$\delta_{i j} x^{i} x^{j}=\mathcal{C}=$ const.

This relation is the same as one which defines embedding of the two-sphere in the three-dimensional euclidean space. The second fact which ensures smoothness is a possibility to define differential calculus. The differential can be written in form of a noncommutative frame [13], with the momenta given by

$p_{a}=\frac{1}{i \hbar} \delta_{a i} x^{i}, \quad a=1,2,3$.

To justify that (2.2) gives a spherical geometry one can either calculate the coordinate components of the metric and obtain the projector to the sphere,

$g^{i j}=e_{a}^{i} e_{b}^{j} \delta^{a b}=\mathcal{C} \delta^{i j}-x^{j} x^{i}$,

or calculate the scalar curvature and obtain a constant. One can also verify that the generators of rotations are Killing vectors, in much the same way se as we later do for de Sitter generators on fuzzy de Sitter space. In addition, there is a well defined commutative limit: the polynomial expansion of an arbitrary matrix $f \in M_{n}$ (taking that $x^{i}$ are in the $n \times n$ irreducible representation),

$f=\sum_{l=0}^{n-1} \frac{1}{l !} f_{a_{1} \ldots a_{l}} x^{a_{1}} \ldots x^{a_{l}}$,

tends in the limit $n \rightarrow \infty$ to the spherical harmonics expansion of the function $f$ on the sphere.

It seems apparent that this simple idea should be easy to implement to other maximally symmetric spaces defined as hyperspheres embedded in higher-dimensional euclidean 
spaces, by using suitable Lie groups and their Casimir relations. It was applied in $[11,14]$ to obtain $2 \mathrm{~d}$ and $4 \mathrm{~d}$ fuzzy hyperboloids: in four dimensions, however, it is more difficult to find the appropriate metric structure. We shall in this section find, within the algebra of de Sitter group $S O(1,4)$, two differential structures which give it a metric of the fourdimensional de Sitter space.

Let us introduce the notation. We have ten generators of the $S O(1,4)$ group $M_{\alpha \beta}, \alpha, \beta=0,1, \ldots 4$; the signature is $\eta_{\alpha \beta}=\operatorname{diag}(-++++)$. The commutation relations are

$\left[M_{\alpha \beta}, M_{\gamma \delta}\right]=i\left(\eta_{\alpha \gamma} M_{\beta \delta}-\eta_{\alpha \delta} M_{\beta \gamma}-\eta_{\beta \gamma} M_{\alpha \delta}+\eta_{\beta \delta} M_{\alpha \gamma}\right)$.

Using $M_{\alpha \beta}$ one can define a vector $W_{\alpha}$ which is quadratic,

$W_{\alpha}=\frac{1}{8} \epsilon_{\alpha \beta \gamma \delta \eta} M^{\alpha \beta} M^{\delta \eta}$,

$\left[M_{\alpha \beta}, W_{\gamma}\right]=i\left(\eta_{\alpha \gamma} W_{\beta}-\eta_{\beta \gamma} W_{\alpha}\right)$.

The $S O(1,4)$ has two Casimir operators:

$\mathcal{Q}=-\frac{1}{2} M_{\alpha \beta} M^{\alpha \beta}, \quad \mathcal{W}=-W_{\alpha} W^{\alpha}$

In order to understand the properties of the algebra with respect to rotations in more detail, we adapt the notation to the $S O(3)$ subgroup and denote the 3 -vector indices by $i, j=1,2,3$. We rename the generators,

$L_{i}=\frac{1}{2} \epsilon_{i j k} M_{j k}, \quad P_{i}=M_{i 4}, \quad Q_{i}=M_{0 i}, \quad R=M_{04}$

The commutation relations of the $S O(1,4)$ are then

$$
\begin{aligned}
& {\left[L_{i}, L_{j}\right]=i \epsilon_{i j k} L_{k}, \quad\left[L_{i}, P_{j}\right]=i \epsilon_{i j k} P_{k},\left[L_{i}, Q_{j}\right]=i \epsilon_{i j k} Q_{k},} \\
& {\left[P_{i}, L_{j}\right]=i \epsilon_{i j k} P_{k}, \quad\left[P_{i}, P_{j}\right]=i \epsilon_{i j k} L_{k},\left[P_{i}, Q_{j}\right]=i \delta_{i j} R,} \\
& {\left[Q_{i}, L_{j}\right]=i \epsilon_{i j k} Q_{k},\left[Q_{i}, P_{j}\right]=-i \delta_{i j} R,\left[Q_{i}, Q_{j}\right]=-i \epsilon_{i j k} L_{k},} \\
& {\left[R, L_{j}\right]=0=i Q_{j},\left[R, P_{j}\right]=i Q_{j}, \quad\left[R, Q_{j}\right]=i P_{j} .}
\end{aligned}
$$

The algebra can be contracted in various ways. Rescaling $P_{i} \rightarrow P_{i} / \sqrt{\Lambda}, R \rightarrow R / \sqrt{\Lambda}$, for $\Lambda \rightarrow 0$ we obtain the Inönü-Wigner contraction to the Poincaré algebra, that is, the flat limit of the de Sitter algebra: $R$ and $P_{i}$ become the generators of four-translations, while $L_{i}$ and $Q_{i}$ generate the 3 -rotations and boosts. In this limit $\mathcal{Q}$ and $\mathcal{W}$ become the Casimir operators of the Poincare algebra: the square of mass and the square of the Pauli-Lubanski vector. Contraction $P_{i} \rightarrow \mu P_{i}, Q_{i} \rightarrow \mu Q_{i}, R \rightarrow \mu^{2} R$ for $\mu \rightarrow \infty$ gives phase space in three dimensions with rotations: $R$ becomes a central element.

In the new notation the components $W_{\alpha}$ are given by

$W_{0}=L_{i} P_{i}=P_{i} L_{i}, \quad W_{4}=-L_{i} Q_{i}=-Q_{i} L_{i}$,

$W_{i}=R L_{i}+\epsilon_{i j k} Q_{j} P_{k}=R L_{i}-\epsilon_{i j k} P_{j} Q_{k}$.

The commutation relations (2.7) can be rewritten as

$$
\begin{array}{lll}
{\left[L_{i}, W_{0}\right]=0,} & {\left[L_{i}, W_{4}\right]=0,} & {\left[L_{i}, W_{j}\right]=i \epsilon_{i j k} W_{k},} \\
{\left[P_{i}, W_{0}\right]=0,} & {\left[P_{i}, W_{4}\right]=-i W_{i},} & {\left[P_{i}, W_{j}\right]=i \delta_{i j} W_{4},} \\
{\left[Q_{i}, W_{0}\right]=-i W_{i},} & {\left[Q_{i}, W_{4}\right]=0,} & {\left[Q_{i}, W_{j}\right]=-i \delta_{i j} W_{0},} \\
{\left[R, W_{0}\right]=-i W_{4},} & {\left[R, W_{4}\right]=-i W_{0},} & {\left[R, W_{j}\right]=0,}
\end{array}
$$

and for the Casimir operators we obtain

$$
\begin{aligned}
-\mathcal{Q} & =-R^{2}-Q_{i} Q_{i}+P_{i} P_{i}+L_{i} L_{i}, \\
-\mathcal{W} & =-\left(W_{0}\right)^{2}+W_{i} W_{i}+\left(W_{4}\right)^{2} \\
& =-\left(W_{0}\right)^{2}-\left[W_{0}, Q_{i}\right]^{2}-\left[W_{0}, R\right]^{2} .
\end{aligned}
$$

Unitary irreducible representations of $S O(1,4)$ are in the notation of [15-17] divided in four classes. In the Class I, the quadratic Casimir operator $\mathcal{Q}>0$ has a continuous range of values and the quartic Casimir operator is zero, $\mathcal{W}=0$. In the Class II representations, $\mathcal{W}=0$, while $\mathcal{Q}$ is discrete, $\mathcal{Q}=$ $-(n-1)(n+2), n=1,2, \ldots$ The Class III representations have continuous $\mathcal{Q}$ and continuous $\mathcal{W}, \mathcal{W}=s(s+1) \mathcal{Q}+$ $(s-1) s(s+1)(s+2), s=1 / 2,1,3 / 2, \ldots .$. Finally, in the Class IV representations both Casimir operators $\mathcal{Q}$ and $\mathcal{W}$ are discrete. Note that Eq. (2.15) implies that if $W_{0}=0$, then the quartic Casimir vanishes, $\mathcal{W}=0$.

Let us discuss possible assignments of coordinates and momenta. The Casimir relation (2.15) directs us to take $W^{\alpha}$ as coordinates of a five-dimensional embedding space, and we shall adopt this identification. The $S O(1,4)$ generators $M_{\alpha \beta}$ as defined are dimensionless, so we introduce

$x^{\alpha}=\ell W^{\alpha}$,

where the constant $\ell$, of dimension of length, will be fixed later. By definition a noncommutative de Sitter space $\mathcal{A}$ is the algebra generated by $x^{\alpha}$ in one of the unitary irreducible representations. The quartic Casimir gives the value of the cosmological constant,

$\eta_{\alpha \beta} x^{\alpha} x^{\beta}=-\ell^{2} \mathcal{W}=\frac{3}{\Lambda}$

The coordinates $x^{\alpha}$ are quadratic in the group generators and therefore they do not close under commutation: in terms of the decomposition (2.12) we have 


$$
\begin{aligned}
{\left[x_{0}, x_{i}\right] } & =i \epsilon_{i j k} \ell x_{j} P_{k}+i \ell L_{i} x_{4}, \\
{\left[x_{4}, x_{i}\right] } & =i \epsilon_{i j k} \ell Q_{j} x_{k}+i \ell L_{i} x_{0}, \\
{\left[x_{0}, x_{4}\right] } & =i \ell L_{i} x_{i},\left[x_{i}, x_{j}\right] \\
& =i \epsilon_{i j k} \ell\left(R x_{k}-P_{k} x_{0}-i Q_{k} x_{4}\right) .
\end{aligned}
$$

These relations are to some extent unsatisfactory because their right-hand-sides contain, besides coordinates, the group generators: if only $x^{\alpha}$ appeared, the interpretation would be much easier. However, this obstruction is not principal: Eqs. $(2.18,2.19)$ show only that calculations, if nontrivial, will not be simplified easily and will possibly depend on the representation.

Momenta have to fulfill a stricter requirement: they must close into an algebra which is at most quadratic. In addition, in order to interpret the frame as a gravitational field,

$g^{\alpha \beta}(x)=e_{A}^{\alpha} e_{B}^{\beta} \eta^{A B}$,

we have to require that the frame elements depend on the coordinates only,

$\left[p_{A}, x^{\alpha}\right]=e_{A}^{\alpha}(x)$

It is thus clear that $W^{\alpha}$ cannot be the momenta as on the fuzzy sphere. A natural choice would be to select momenta among the group generators. If we wish to preserve the full de Sitter symmetry we shall choose as momenta all $M_{\alpha \beta}$,

$i p_{A}=\sqrt{\Lambda} M_{\alpha \beta}$,

where the index $A, A=1, \ldots 10$, denotes antisymmetric pairs $[\alpha \beta]$. To get dimensions right we introduced in the last formula $\sqrt{\Lambda}$; we systematically use convention $\hbar=1$. The square root of the cosmological constant in (2.22) is in fact implied by the frame formalism as, when the momenta form a Lie group,

$\left[p_{A}, p_{B}\right]=C_{A B}^{D} p_{D}$

the curvature scalar is quadratic in the structure constants,

$R=\frac{1}{4} C^{A B D} C_{D A B}$

To be completely accurate we should in fact have put

$i p_{A}=\sqrt{\zeta \Lambda} M_{\alpha \beta}$,

then the normalization of the scalar curvature, $R=4 \Lambda$, would have given $\zeta=1 / 3$; we will, however, it being simpler, keep (2.22). The constant $\ell$ we fix as $\ell=k \sqrt{\Lambda}, \quad \mathcal{W}=-\frac{3}{k^{2} \Lambda^{2}}$,

where $k$ is the scale of noncommutativity of dimension length squared. In consequence $k$ enters only in the position commutators,

$\left[x_{0}, x_{i}\right]=\hbar\left(\epsilon_{i j k} x_{j} p_{k+3}+p_{i} x_{4}\right)$,

$\left[x_{4}, x_{i}\right]=k\left(\epsilon_{i j k} p_{j+6} x_{k}+p_{i} x_{0}\right)$,

$\left[x_{0}, x_{4}\right]=\hbar p_{i} x_{i}$,

$\left[x_{i}, x_{j}\right]=\hbar \epsilon_{i j k}\left(p_{10} x_{k}-p_{k+3} x_{0}-p_{k+6} x_{4}\right)$.

Therefore the commutative limit is defined as $k \rightarrow 0$.

The given choice of the differential structure might seem at first sight unusual: we have spacetime of $5-1=4$ dimensions, with the tangent space of 10 dimensions. As we explained earlier, this comes formally with noncommutativity of coordinates. To understand the meaning of the introduced differential $d$ we proceed to the metric and the laplacian. Denoting

$i p_{i}=\sqrt{\Lambda} L_{i}, \quad i p_{i+3}=\sqrt{\Lambda} P_{i}, \quad i p_{i+6}=\sqrt{\Lambda} Q_{i}$
$i p_{10}=\sqrt{\Lambda} R, \quad i=1,2,3$

and introducing a locally flat metric $g^{A B}=\eta^{A B}$ with signature $(++++++----)$, we obtain the following expressions for the frame components, $e_{A}^{\alpha}=\left[p_{A}, x^{\alpha}\right]$ :

$e_{j}^{0}=0, \quad e_{j+3}^{0}=0 \quad e_{j+6}^{0}=\sqrt{\Lambda} x^{j} \quad e_{10}^{0}=\sqrt{\Lambda} x^{4}$,

$e_{j}^{i}=-\epsilon_{j k}^{i} \sqrt{\Lambda} x^{k} \quad e_{j+3}^{i}=\delta_{j}^{i} \sqrt{\Lambda} x^{4}$

$e_{j+6}^{i}=\delta_{j}^{i} \sqrt{\Lambda} x^{0} \quad e_{10}^{i}=0$,

$e_{j}^{4}=0, \quad e_{j+3}^{4}=-\sqrt{\Lambda} x^{j} \quad e_{j+6}^{4}=0 \quad e_{10}^{4}=\sqrt{\Lambda} x^{0}$.

From these expressions we can find differentials $\mathrm{d} x^{\alpha}=$ $e_{A}^{\alpha} \theta^{A}$

$\mathrm{d} x^{0}=\sqrt{\Lambda} x^{i} \theta^{i+6}+\sqrt{\Lambda} x^{4} \theta^{10}$,

$\mathrm{d} x^{i}=-\epsilon^{i}{ }_{j k} \sqrt{\Lambda} x^{k} \theta^{j}+\sqrt{\Lambda} x^{4} \theta^{j+3}$ $+\sqrt{\Lambda} x^{0} \theta^{j+6}$

$\mathrm{d} x^{4}=-\sqrt{\Lambda} x^{i} \theta^{i+3}+\sqrt{\Lambda} x^{0} \theta^{10}$.

The spacetime components of the metric, $g^{\alpha \beta}=e_{A}^{\alpha} e_{B}^{\beta} \eta^{A B}$, $\alpha=0,1,2,3,4$, are 
$g^{\alpha \beta}=\Lambda\left(\begin{array}{ccc}-\left(x^{i}\right)^{2}-\left(x^{4}\right)^{2} & -x^{i} x^{0} & -x^{4} x^{0} \\ -x^{0} x^{j} & \left(-\left(x^{0}\right)^{2}+\left(x^{i}\right)^{2}+\left(x^{4}\right)^{2}\right) \delta^{i j}-x^{j} x^{i} & -x^{4} x^{j} \\ -x^{0} x^{4} & -x^{i} x^{4} & -\left(x^{0}\right)^{2}+\left(x^{i}\right)^{2}\end{array}\right) ;$

this can be simplified to

$g^{\alpha \beta}=3 \eta^{\alpha \beta}-\Lambda x^{\beta} x^{\alpha}$.

In the commutative limit $g^{\alpha \beta}$ is singular and reduces to the projector on the four-dimensional de Sitter space defined by (2.15); it projects out the radius vector $x_{\alpha}$. When we calculate the curvature which corresponds to the given frame, as shown in the appendix, we obtain $R=12 \Lambda$; more generally, using rescaling (2.25) we have

$R=12 \zeta \Lambda$

Quadratic Casimir operator $\mathcal{Q}$ is usually related to mass $[20,21]$. For a scalar field $\Phi(x)$ the laplacian and the KleinGordon equation are written as

$\Delta \Phi=-\eta^{A B} e_{A} e_{B} \Phi=-\eta^{A B}\left[p_{A},\left[p_{B}, \Phi\right]\right]=m^{2} \Phi$,

and clearly mass corresponds to the value(s) of $\mathcal{Q}$ in the adjoint representation. In the contraction limit to the Poincaré group, generators $L_{i}$ and $Q_{i}$ become negligible so the mass (2.14) reduces to

$-\left.\mathcal{Q}\right|_{\Lambda \rightarrow 0}=-R^{2}+\left(P_{i}\right)^{2}$.

It possible to reduce the number of momenta and the dimensionality of the tangent space while keeping the metric of $\mathcal{A}$ de Sitter. We introduce another set of momenta

$i \tilde{p}_{0}=\sqrt{\Lambda} R, \quad i \tilde{p}_{i}=\sqrt{\Lambda}\left(P_{i}+Q_{i}\right), \quad i=1,2,3$

and denote the correspondingly defined differential by $\tilde{d}$; coordinates are the same. The momentum commutators are

$\left[\tilde{p}_{0}, \tilde{p}_{i}\right]=-\sqrt{\Lambda} \tilde{p}_{i}, \quad\left[\tilde{p}_{i}, \tilde{p}_{j}\right]=0$.

Applying the algebra relations we get now for the frame elements

$$
\begin{aligned}
& \tilde{e}_{0}^{0}=\left[\tilde{p}_{0}, x^{0}\right]=-x^{4}, \quad \tilde{e}_{0}^{i}=\left[\tilde{p}_{0}, x^{i}\right]=0, \\
& \tilde{e}_{0}^{4}=\left[\tilde{p}_{0}, x^{4}\right]=x^{0}, \quad \tilde{e}_{j}^{0}=\left[\tilde{p}_{j}, x^{0}\right]=x^{j}, \\
& \tilde{e}_{j}^{i}=\left[\tilde{p}_{j}, x^{i}\right]=\delta_{i j}\left(x^{0}+x^{4}\right), \quad \tilde{e}_{j}^{4}=\left[\tilde{p}_{j}, x^{4}\right]=-x^{j} .
\end{aligned}
$$

Therefore the coordinate differentials are given by

$\tilde{\mathrm{d}} x^{0}=x^{4} \tilde{\theta}^{0}+x^{j} \tilde{\theta}^{j}$,

$\tilde{\mathrm{d}} x^{i}=\left(x^{0}+x^{4}\right) \tilde{\theta}^{i}$,

$\tilde{\mathrm{d}} x^{4}=x^{0} \tilde{\theta}^{0}-x^{j} \tilde{\theta}^{j}$,

and we easily recognize that variable $x^{0}+x^{4}$ should be introduced as a new coordinate, time. Denoting

$\tau=-\log \left(x^{0}+x^{4}\right)$

we find

$\mathrm{d}\left(x^{0}+x^{4}\right)=\left(x^{0}+x^{4}\right) \tilde{\theta}^{0}, \quad \tilde{\theta}^{0}=-\mathrm{d} \tau$.

The line element becomes in the classical limit that of de Sitter space,

$\tilde{\mathrm{d}} s^{2}=-\left(\tilde{\theta}^{0}\right)^{2}+\left(\tilde{\theta}^{i}\right)^{2}=-\tilde{\mathrm{d}} \tau^{2}+e^{2 \tau} \tilde{\mathrm{d}} x^{i} \tilde{\mathrm{d}} x^{i}$.

The remaining differential $\tilde{\mathrm{d}} x^{4}$ is not independent: its value follows from the Casimir constraint $\tilde{\mathrm{d}}\left(x^{\alpha} x_{\alpha}\right)=0$. Assuming that the metric in frame components has the signature $(-+$ ++ ) for the coordinate components we obtain

$\tilde{g}^{\alpha \beta}=\left(\begin{array}{ccc}-\left(x^{4}\right)^{2}+\left(x^{i}\right)^{2} & x^{i}\left(x^{4}+x^{0}\right) & -x^{4} x^{0}-\left(x^{i}\right)^{2} \\ x^{i}\left(x^{4}+x^{0}\right) & \delta^{i j}\left(x^{4}+x^{0}\right)^{2} & -x^{i}\left(x^{4}+x^{0}\right) \\ -x^{4} x^{0}-\left(x^{i}\right)^{2} & -x^{i}\left(x^{4}+x^{0}\right) & -\left(x^{0}\right)^{2}+\left(x^{i}\right)^{2}\end{array}\right)$.

Again this is a projector to the hypersphere which projects out $x_{\alpha}$.

We achieved to obtain four-dimensional tangent space. The Laplace operator induced by $\tilde{d}$ is given by

$\tilde{\Delta} \Phi=\Lambda[R,[R, \Phi]]-\Lambda\left[P_{i}+Q_{i},\left[P_{i}+Q_{i}, \Phi\right]\right]=\tilde{m}^{2} \Phi$,

and although it is not invariant under the full de Sitter group, it has the correct Poincaré limit under the contraction,

$-\left.\tilde{m}^{2}\right|_{\Lambda \rightarrow 0}=-R^{2}+\left(P_{i}\right)^{2}$.

The laplacian $\tilde{\Delta}$ invariant under the 3-rotations and boosts,

$\left[L_{j},-R^{2}+\left(P_{i}\right)^{2}\right]=0, \quad\left[Q_{j},-R^{2}+\left(P_{i}\right)^{2}\right]=0$. 
As shown in the appendix, the scalar curvature related to $\tilde{d}$ is constant,

$\tilde{R}=\frac{3}{4} \Lambda$,

and if we wish to have the usual value $4 \Lambda$ for the scalar curvature we need to redefine the momenta, $\tilde{p}_{\alpha} \rightarrow \tilde{\zeta} \tilde{p}_{\alpha}$ with $\tilde{\zeta}=16 / 3$. The fact that $\tilde{\Delta}$ is not invariant under the full de Sitter group indicates that the metric is not invariant too: indeed this is the case and it can be confirmed by an analysis of the Killing equations which is done in the appendix.

\section{Noncommutative de Sitter space, II}

To construct a four-dimensional de Sitter space we started in the previous section from the algebra of de Sitter group $S O(1,4)$. Within this algebra we identified coordinates, momenta and the moving frame consistently with rules imposed by the frame formalism: as result we found two natural realizations of fuzzy de Sitter space. A drawback of this procedure is the existence of a number of operators which have no direct physical interpretation, while they cannot be avoided in calculations: one would prefer to have an algebra which is as small as possible, minimal.

One method to search for such an algebra is to try to construct it: first, to assume that it exists by making an Ansatz for the commutation relations; one needs a further Ansatz for the moving frame, the one that gives the required form of the metric. Using these two premises one solves the constraints: the Jacobi, the frame and the compatibility equations. Such programme can be systematically done only approximately, in linear order in noncommutativity (otherwise, in the course of calculations one would have to use the multiplication rules which one is looking for) and as result one obtains a noncommutative spacetime, that is, an algebra and a calculus. Following this approach we have made in our previous papers a survey of noncommutative algebras generated by four, five and six elements, [8-10]. The original motivation was in part to use interior derivatives, thereby decreasing the dimension of phase space. The summary of the results dimensionwise is as follows. The lowest-dimensional spacetime which can be constructed has four generators (the corresponding phase space has five), but the metric is necessarily nonstatic: it is in fact unique. When we add one more generator, that is, within the set of five-dimensional position algebras, the constraints get considerably relaxed and we obtain a large family of static spherically symmetric noncommutative geometries.

We wish here to generalize the frame Ansatz and the constraints of [8] in order to obtain noncommutative cosmological spacetimes of the Friedmann-Robertson-Walker type. Let us briefly introduce the notation and write down the equa- tions; for all technical details we refer to [8] as calculations are to some extent analogous. Coordinates are denoted by

$x^{0}=t, \quad x^{a}=\rho \xi^{a}, \quad x^{4}=r, \quad a=1,2,3$.

Normalized vector $\xi^{a}$ describes two angular variables, polar and azimuthal angle; radial coordinate (measuring either the area of the sphere or the radial distance) is $r$, time is $t$. The $x^{a}$ satisfy

$x_{a} x^{a}=\rho^{2}$,

therefore the additional fifth coordinate $\rho$ is analogous to a radius but of an additional, extra dimension. The $\xi^{a}$ are the generators of the $S O(3)$ algebra in the irreducible $n \times n$ representation: the momenta $p_{a}$ are taken to be proportional to $\xi^{a}$, as on the fuzzy sphere.

We assume that the form of the position algebra $\mathcal{A}$ is

$\left[\xi^{a}, \xi^{b}\right]=\frac{2 i}{n} \epsilon^{a b c} \xi_{c}, \quad\left[\xi^{a}, \rho\right]=\left[\xi^{a}, r\right]=\left[\xi^{a}, t\right]=0$,

$[\rho, t]=i \hbar J^{0} \rho, \quad[r, t]=i \hbar J$,

$[\rho, r]=i \hbar J^{4} \rho$,

that is, a tensor product $s o(3) \otimes \mathcal{A}^{\prime}$ where $\mathcal{A}^{\prime}$ is the algebra generated by $r, t$, and $\rho$. Differential calculus mixes the two subalgebras: the Ansatz for the frame is given by

$$
\begin{array}{ll}
\theta^{a}=-h \rho^{-1} \epsilon_{b c}^{a} x^{b} d x^{c}+\rho^{-2} x_{b} \theta^{b} x^{a}, & \mathrm{~d} x^{a}=(h \rho)^{-1} \epsilon_{b c}^{a} x^{b} \theta^{c}, \\
\theta^{4}=g \mathrm{~d} r, & \mathrm{~d} r=g^{-1} \theta^{4}, \\
\theta^{0}=f \mathrm{~d} t+k x^{a} \theta^{a}, & \mathrm{~d} t=f^{-1} \theta^{0}-k f^{-1} x^{a} \theta^{a} .
\end{array}
$$

This Ansatz implies, as on the fuzzy sphere,

$\rho \mathrm{d} \rho+\mathrm{d} \rho \rho=0$,

that is, $\rho$ is in the commutative limit a constant. To impose spherical symmetry we assume that $f, g, h$, and $k$, as well as $J, J_{0}$, and $J_{4}$ are functions of $\rho, r$, and $t$ only. We thus have seven unknown functions, which are to be determined from the constraint equations. We will consider here only the case $k=0$, which gives the usual diagonal form of the metric; $k \neq 0$ allows one to extend the construction to the generalized Taub-NUT spaces.

Consistency of the frame with the algebra, the Jacobi, and the frame constraints gives the following set of equations:

$$
\begin{array}{ll}
\dot{J}^{4}=0, \quad J^{0 \prime}=0, & h^{\prime} J^{4}+\dot{h} J^{0}=0, \\
J^{4 \prime}+g^{-1}\left(g^{\prime} J^{4}+\dot{g} J^{0}\right)=0, & \left(h+\rho \partial_{\rho} h\right) J^{4}-\dot{h} J=0, \\
J^{0 \prime}+f^{-1}\left(f^{\prime} J^{4}+\dot{f} J^{0}\right)=0, & \left(h+\rho \partial_{\rho} h\right) J^{0}+h^{\prime} J=f^{-1} k h^{2} \rho^{2}, \\
\dot{J}+f^{-1}\left(\dot{f} J-\rho \partial_{\rho} f J^{4}\right)=0, & J^{\prime}+g^{-1}\left(g^{\prime} J+\rho \partial_{\rho} g J^{0}\right)=0, \\
k^{\prime} J^{4}+\dot{k} J^{0}=0, & \left(k+\rho \partial_{\rho} k\right) J^{4}-\dot{k} J=0,
\end{array}
$$


where $\dot{f}=\partial_{t} f$ and $f^{\prime}=\partial_{r} f$. The equations are coupled and nonlinear, and thus relatively complicated; but we do not need to solve them in full generality because of the diffeomorphism invariance of the formalism, which can be used to fix some of the variables. We choose the radial coordinate such that the area of sphere be $4 \pi r^{2}$, that is, we put $h=r$. We assume further that the metric has the Schwarzschild form, $f g=1$. In the static case, when no function depends on time, we find a solution

$J^{4}=0, \quad J^{0}=J^{0}(\rho), \quad J=-r J_{0}(\rho)$,

$h=r, \quad f=\frac{1}{g}=\gamma r F(\rho r)$,

the function $F$ can be an arbitrary function of its argument. The corresponding metric in the commutative limit has the line element

$\mathrm{d} s^{2}=-f^{2} \mathrm{~d} t^{2}+\frac{1}{f^{2}} \mathrm{~d} r^{2}+r^{2} \mathrm{~d} \Omega$.

Though (3.10) is restricted in its form, it gives a large family of solutions. We have seen that in the classical limit $\rho$ becomes a constant: therefore taking for example

$F(\rho r)=\sqrt{\frac{1-\rho^{2} r^{2}}{\rho^{2} r^{2}}}$

and identifying $\rho$ with the cosmological constant,

$\rho^{2}=\gamma^{2}=\frac{\Lambda}{3}$,

we obtain the de Sitter metric in static coordinates. As a consequence, we find that the cosmological constant is quantized.

The noncommutative de Sitter space $\mathcal{A}$ is in this version a relatively small algebra, generated by five elements; it is, in contrast to the spacetime found in the previous section, closed. But to obtain the phase space we have to extend it by one generator, $p_{4}$. Namely from (3.5) and our solution we see that the momentum $p_{4}$ has to satisfy

$\left[p_{4}, t\right]=0, \quad\left[p_{4}, x^{a}\right]=0, \quad\left[p_{4}, \rho r\right]=0$,

and therefore it does not belong to $\mathcal{A}$. These relations can be solved, not uniquely, within a larger algebra.

In order to find within this same framework the FriedmannRobertson-Walker type solutions, we should include the dependence on time. It is necessary also to take $J^{4} \neq 0$. Again, instead of attempting to find the most general solution we seek a particular one, of the form

$f=1, \quad g=a(t) G(r, \rho), \quad h=a(t) r$.
We obtain the following set of equations:

$\partial_{t} J^{0}=0$

$$
\partial_{t} J^{4}=0,
$$

$\partial_{t} J=0$,

$\partial_{r} J^{0}=0$,

$a J^{4}+\dot{a} J^{0}=0$,

$a J^{4}-\dot{a} J=0$,

$\partial_{r} J J^{4}-\partial_{r} J_{4} J-\rho \partial_{\rho} J^{4} J^{0}+\rho \partial_{\rho} J^{0} J^{4}=0$.

These equations have a solution similar to (3.8),

$J^{0}=J^{0}(\rho), \quad J=-r J^{0}, \quad J^{4}=-\frac{\dot{a}}{a} r J^{0}$.

The remaining constraint gives $G(r, \rho)=\rho$, so we find for the frame

$f=1, \quad g=a(t) \rho, \quad h=a(t) r$.

The scale factor $a(t)$ can be arbitrary; the limiting classical metric is given by

$\mathrm{d} s^{2}=-\mathrm{d} t^{2}+a^{2}(t)\left(\rho^{2} \mathrm{~d} r^{2}+r^{2} \mathrm{~d} \Omega\right)$,

where $\rho$ is as before constant so we can choose $\rho=1$. Taking for example the exponential function,

$a(t)=\exp \sqrt{\frac{\Lambda}{3}} t$,

we obtain the de Sitter space in the FRW form. In general, we find a family of fuzzy Friedmann-Robertson-Walker geometries.

\section{Conclusions}

We constructed in this paper, using the noncommutative frame formalism, essentially two different versions of a noncommutative four-dimensional de Sitter space. Let us review and compare them.

The de Sitter spaces constructed in Sect. 2 are based on the algebra of the de Sitter group $S O(1,4)$. Coordinates which generate spacetime $\mathcal{A}$ are proportional to the 5-vectors $W^{\alpha}$ (2.6), so in the unitary irreducible representations of the group, the Casimir relation (2.15) defines four-dimensional de Sitter space as an embedding in five dimensions. The value of the quartic Casimir operator is related to the cosmological constant $\Lambda$ and noncommutativity scale $k,(2.26)$; whether $\Lambda$ has a discrete or a continuous spectrum depends on the representation. Spacetime $\mathcal{A}$ has the somewhat unpleasant property that the coordinates do not close under commutation. But as is explained in the text, this aspect is not essential: what is really necessary (and sufficient) for a physical interpretation is that commutators with momenta (2.21), which give the frame and the metric, can be written in terms of coordinates 
solely. Within $\mathcal{A}$ we formulate two differential calculi, both based on the generators of the de Sitter group, $M_{\alpha \beta}$ : of course, other differential structures can be constructed but they give other geometries. The first calculus has as momenta all group generators and therefore the corresponding tangent space is 10-dimensional. The second calculus has four momenta and the tangent space, which is four-dimensional. In both cases the metric in coordinate components $(2.20)$ is a $5 \times 5$ matrix which projects to the four-dimensional de Sitter space $\mathcal{A}$; also, the scalar curvature is in both cases constant. The two calculi have different laplacians: the laplacian $\Delta$ is related to the quadratic Casimir of the $S O(1,4)$, which is in field theories on the commutative de Sitter space usually related to the mass. The second laplacian $\tilde{\Delta}$ is not invariant under the full de Sitter symmetry, but it is invariant under the Lorentz subgroup. We therefore obtain two different Klein-Gordon equations: in both cases the Inönü-Wigner contraction gives the usual mass.

Section 3 contains two more versions of a fuzzy de Sitter space, one in the static and another in the FRW coordinates: we can obtain in fact noncommutative versions of all Friedmann-Robertson-Walker spacetimes. The strategy of the calculation is in Sect. 3 inverted: instead of fixing the algebra in advance we search for it, and therefore the position space $\mathcal{A}$ which we obtain is always, by definition, closed. However, it is not minimal: it has $6-1=5$ generators; see Eq. (3.2). Although the additional variable $\rho$ is not a Casimir operator, it is constant in the sense that its differential is zero, $\mathrm{d}\left(\rho^{2}\right)=0$. We interpret $\rho$ as the size of the additional dimension: classically its value is related, in the cosmological case, to $\Lambda$ and $k$; see Eq. (3.12). We have not solved, within this approach, for the full phase space algebra and therefore the laplacians are missing: equations for $p_{\alpha}$ are, however given; see (3.13). One of the problems with searching for algebras which satisfy equations like (3.13) is that there are 'too many' zeros on the right-hand sides, and consequently too many extensions are possible: it is much easier to solve the Jacobi identities when they contain nonsingular expressions. This presents also the main technical difference between the two approaches presented. On the one hand, the a priori choice of the algebra of the $S O(1,4)$ group done in Sect. 2 might seem to be quite arbitrary: in principle, one would prefer to have a set of equations and to find a unique solution to them. But on the other hand it is hard to imagine that, starting from Eqs. (2.40), one would arrive at the de Sitter algebra: there are many more 'equally good' algebras which contain these equations.

In fact, the algebra of the $S O(1,4)$ (or the $S O(2,3)$ ) group seems to be the ideal framework for noncommutative four-dimensional geometries. It is 'big enough' to be a phase space: the number of its generators is $10-2=8$. Also, the existence of three independent 3 -vectors and one scalar gives enough room to construct rotationally invariant spaces: this is seemingly impossible within the algebra of $S O(1,3)$. Among various versions of fuzzy de Sitter space here analyzed, the one based on the de Sitter algebra with the four-dimensional tangent space is perhaps the most appealing, because of its dimension. It has an additional merit: it can be generalized to arbitrary even-dimensional space. Namely, starting from the $S O(1, n)$ group and its generators $M_{\alpha \beta}$, $\alpha, \beta=0,1, \ldots n$, for even $n$ there is a vector

$W^{\alpha}=\epsilon^{\alpha \alpha_{1} \ldots \alpha_{n}} M_{\alpha_{1} \alpha_{2}} \ldots M_{\alpha_{n-1}, \alpha_{n}}$.

The square of $W^{\alpha}$ is the Casimir operator of the highest rank in $S O(1, n)$. The corresponding Casimir relation defines an embedding of the $n$-dimensional hypersphere in a $(n+1)$ dimensional space. By analogy with the four-dimensional case, the choice of

$i \tilde{p}_{0}=M_{0 n}, \quad i \tilde{p}_{i}=M_{0 i}+M_{i n}, \quad i=1,2, \ldots n-1$,

as momenta gives the $n$-dimensional tangent space. We find therefore $n$-dimensional fuzzy de Sitter spacetime in coordinates which give the flat sections, together with the corresponding time-dependent metric.

The other fuzzy de Sitter space based on the $S O(1,4)$ group, with 10-dimensional tangent space, is interesting because it preserves the full de Sitter symmetry. Somewhat counterintuitive is a large discrepancy of the dimensionalities of spacetime and tangent space, 4 and 10; though as we discussed earlier, equality of these two numbers is hardly to be expected. There are many physical arguments which support the intuition that at small scales coordinates are noncommuting operators. One can develop a similar intuition regarding the linear space of differentials: namely, if differentials are in some way related to the fluctuations of coordinates, there is no reason why they cannot have 'more dimensions' than coordinates themselves: after all, fluctuations depend on the states of the system too. Similarly, one can argue that a coordinate restricted to the 'subspace of a noncommutative manifold' can fluctuate, besides tangent, in orthogonal directions too. The difference in dimensions which we have here and in other cases [13] gives a completely different counting of the degrees of freedom of fields on a noncommutative space and it can potentially give very interesting models.

In any case, both $S O(1,4)$-based models of fuzzy de Sitter space can be very useful. Their unitary irreducible representations are known, and that gives many possibilities for further investigation: one can for example discuss the spectra of the coordinates, coherent states, field theories etc. An equally important task would be to obtain perturbations as in [22] for perturbations of the flat space. Finally, fuzzy de Sitter space proves again that the noncommutative frame formalism is a good and systematic method to describe noncommutative 
geometries, but also that we need more work to explore all its properties in order to use it properly.

Acknowledgments This work was supported by the Serbian Ministry of Education, Science and Technological Development Grant ON171031. The authors worked on the subject while visiting AEI in Berlin and they would like to thank Hermann Nicolai for his hospitality.

Open Access This article is distributed under the terms of the Creative Commons Attribution 4.0 International License (http://creativecomm ons.org/licenses/by/4.0/), which permits unrestricted use, distribution, and reproduction in any medium, provided you give appropriate credit to the original author(s) and the source, provide a link to the Creative Commons license, and indicate if changes were made.

Funded by SCOAP ${ }^{3}$.

\section{Appendix}

We give technical details related to the calculations and results given in Sect. 2; for a more rigorous introduction we refer to [7]. Both differential calculi considered in Sect. 2 have momenta which close into a Lie algebra. In this case properties of the space of $p$-forms simplify: one can show for example that the frame forms anticommute, $\theta^{A} \theta^{B}+\theta^{B} \theta^{A}=$ 0 , which is not the case in general. The expression for the scalar curvature is also very simple, see Eq. (2.24), so in order to calculate it we only need to determine the structure constants.

If we take as momenta all de Sitter group generators $p_{A}$, using the enumeration (2.29) we find that nonvanishing $C_{A B C}$ are

$$
\begin{aligned}
& C_{i j k}=\epsilon_{i j k}, \quad C_{i, j+3, k+3}=\epsilon_{i j k}, \\
& \quad C_{i, j+6, k+6}=-\epsilon_{i j k}, \quad C_{i+3, j+6,10}=-\delta_{i j} .
\end{aligned}
$$

Nonzero are also all permutations of the structure constants with the given indices, and $C_{A B C}$ are completely antisymmetric. Using (5.1) one can easily obtain (2.37). For the second set of momenta $\tilde{p}_{\alpha}$ the nonvanishing structure constants are

$\tilde{C}_{i 0 j}=-\tilde{C}_{i j 0}=\delta_{i j}$

These constants are neither cyclic nor fully antisymmetric; they give, however, a constant curvature scalar, (2.53).

The antisymmetry of the structure constants is important when we calculate the Lie derivative of the metric. The Lie derivative, with its usual properties, can in general be defined on noncommutative spaces [7]; we will use it here to check whether derivations defined by the group generators are also the Killing vectors. The interior product of a vector field $X$ and a 1 -form $\eta$ is given by

$i_{X} \eta=\eta(X)$.
The action of the Lie derivative $\mathcal{L}_{X}$ can be extended, from the action on functions,

$\mathcal{L}_{X} f=X f$

to the action on 1-forms and their products by linearity, the Leibniz rule, and the formula

$\mathcal{L}_{X}=i_{X} d+d i_{X}$

In particular, using the fact that the frame components of the metric are constants,

$g\left(\theta^{A} \otimes \theta^{B}\right)=\eta^{A B}$

we find how $\mathcal{L}_{X}$ acts on the metric:

$$
\begin{aligned}
\left(\mathcal{L}_{X} g\right)\left(\theta^{A} \otimes \theta^{B}\right)= & -g\left(\mathcal{L}_{X} \theta^{A} \otimes \theta^{B}\right) \\
& -g\left(\theta^{A} \otimes \mathcal{L}_{X} \theta^{B}\right) .
\end{aligned}
$$

That is, in order to check whether the vector field $X$ is a Killing vector,

$\mathcal{L}_{X} g=0$,

we need only to determine $\mathcal{L}_{X} \theta^{A}$ and apply (5.7). Using Eq. (5.5) we can easily find the Lie derivatives corresponding to the frame vectors $e_{A}$,

$\mathcal{L}_{e_{A}} \theta^{B}=-C^{B}{ }_{A D} \theta^{D}$.

We thus have, in the case of the first calculus,

$\left(\mathcal{L}_{e_{A}} g\right)\left(\theta^{B} \otimes \theta^{D}\right)=C^{B}{ }_{A}^{D}+C^{D}{ }_{A}^{B}=0$,

that is, all derivations $e_{A}$ are the Killing vectors and spacetime has the de Sitter group as its symmetry. For the second calculus this is not the case: we have for example

$\left(\mathcal{L}_{\tilde{e}_{0}} g\right)\left(\theta^{i} \otimes \theta^{j}\right)=2 \delta^{i j}, \quad\left(\mathcal{L}_{\tilde{e}_{i}} g\right)\left(\theta^{0} \otimes \theta^{j}\right)=\delta^{i j}$,

so neither $\tilde{e}_{0}$ nor $\tilde{e}_{i}$ are Killing vectors. Rotations, however, are a symmetry of the metric,

$\mathcal{L}_{X_{i}} g=0$,

where $X_{i} f=\left[L_{i}, f\right]$. This can be confirmed by extrapolating Eq. (5.9) to the generators of rotations using (5.5) and the expansions (2.44) and (2.47). 


\section{References}

1. J. Heckman, H. Verlinde, Nucl. Phys. B 894, 58 (2015). arXiv:1401.1810 [hep-th]

2. Freidel, L., Leigh, R.G., Minic, D.: JHEP 1506, 006 (2015). arXiv:1502.08005 [hep-th]

3. R. Blumenhagen, M. Fuchs, F. Hassler, D. Lust, R. Sun, JHEP 1404, 141 (2014). arXiv:1312.0719 [hep-th]

4. P. Aschieri, M. Dimitrijevic, F. Meyer, J. Wess, Class. Quant. Grav. 23, 1883 (2006). arXiv:hep-th/0510059

5. J. Wess, J, Gen. Rel. Grav. 39, 1121 (2007). arXiv:hep-th/0607251

6. N. Chandra, H.W. Groenewald, J.N. Kriel, F.G. Scholtz, S. Vaidya, J. Phys. A 47( 44), 445203 (2014). arXiv:1407.5857 [hep-th]

7. J. Madore, An introduction to noncommutative differential geometry and its physical applications. Lond. Math. Soc. Lect. Note Ser. 257 (2000)

8. M. Buric, J. Madore, Eur. Phys. J. C 74, 2820 (2014). arXiv: 1401.3652 [hep-th]

9. M. Buric, J. Madore, Int. J. Mod. Phys. A 24, 2783 (2009)

10. M. Buric, J. Madore, Eur. Phys. J. C 58, 347 (2008). arXiv:0807.0960 [hep-th]
11. J.P. Gazeau, J. Mourad, J. Queva. arXiv:quant-ph/0610222

12. J.P. Gazeau, F. Toppan, Class. Quant. Grav. 27, 025004 (2010). arXiv:0907.0021 [hep-th]

13. J. Madore, Class. Quant. Grav. 9, 69 (1992)

14. D. Jurman, H. Steinacker, JHEP 1401, 100 (2014). arXiv: 1309.1598 [hep-th]

15. L.H. Thomas, Ann. Math. 113 (1941)

16. T.D. Newton, Ann. Math. 730 (1950)

17. J. Dixmier, Bull. Soc. Math. France 89, 9 (1961)

18. E. Joung, J. Mourad, R. Parentani, JHEP 0608, 082 (2006). arXiv:hep-th/0606119

19. E. Joung, J. Mourad, R. Parentani, JHEP 0709, 030 (2007). arXiv:0707.2907 [hep-th]

20. T. Garidi. arXiv:hep-th/0309104

21. J.P. Gazeau, M. Lachieze Rey, PoS IC 2006, 007 (2006). arXiv:hep-th/0610296

22. M. Buric, T. Grammatikopoulos, J. Madore, G. Zoupanos, JHEP 0604, 054 (2006). arXiv:hep-th/0603044 